\title{
Rate-splitting for the Deterministic Broadcast Channel
}

\author{
Todd P. Coleman, Michelle Effros, Emin Martinian, and Muriel Médard \\ colemant@mit.edu, effros@caltech.edu,emin@alum.mit.edu,medard@mit.edu \\ MIT, Caltech, Mitsubishi Electronic Research Labs, MIT
}

\begin{abstract}
We show that the deterministic broadcast channel, where a single source transmits to $M$ receivers across a deterministic mechanism, may be reduced, via a rate-splitting transformation, to another $(2 M-1)$-receiver deterministic broadcast channel problem where a successive encoding approach suffices. Analogous to rate-splitting for the multiple access channel and source-splitting for the Slepian-Wolf problem, all achievable rates (including non-vertices) apply. This amounts to significant complexity reduction at the encoder.
\end{abstract}

\section{INTRODUCTION}

The structural similarity between the capacity regions and random coding achievable rate strategies for the Slepian-Wolf (SW) problem [1] and the deterministic broadcast channel (DBC) problem [2], [3] has been illustrated in [4, sec. III]. Duality connections between the capacity regions of the discrete multiple access channel (DMAC) problem [5], [6] and the DBC have been recently discussed in [7]. Also, a duality between the random coding achievable rate strategies for the DMAC and SW problem has been discussed in [8, p. 416-418]. Different aspects of these dualities have also been explored in [9], [10]. This suggests the existence of a strong relationship amongst these three canonical information theory problems.

Practically speaking, the decoding process is one of the biggest challenges in achieving rates near the boundary of the achievable region in the SW and DMAC problems, as a single decoder must jointly decode messages from multiple senders. Analogously, the encoding process presents itself as a formidable task for the DBC problem, as one encoder must jointly incorporate multiple messages into a single channel codeword.

Recently, 'rate-splitting' has been discussed [11] as a way to significantly reduce the complexity of decoding in the DMAC, for any achievable rate - not just a 'vertex' or 'corner point'. This technique decomposes the problem of jointly decoding $M$ users into a set of $2 M-1$ pipelined single-user channel decoding with side information problems, where each user must be split at most once. Similarly, 'source-splitting' has been discussed [12], [13], [14] as a practical way to decode for the SW problem. This technique similarly decomposes the problem of jointly decoding $M$ sources into a set of $2 M-1$ pipelined single-source decoding with side information [15] problems, where each source must be split at most once.

Motivated by the strong relationship between the SW, DBC and DMAC problems along with the splitting techniques for the SW and DMAC problems, we consider here a rate-splitting

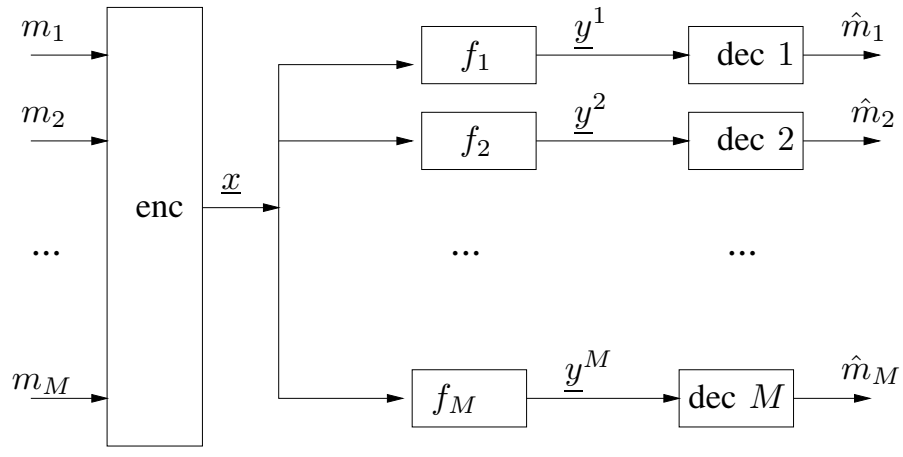

Fig. 1. Basic Model for the Deterministic Broadcast Channel.

technique for the DBC. Here we illustrate that we may take an arbitrary point in the $M$-receiver DBC achievable rate region and transform it, by rate-splitting each source at most once, to a vertex of another $2 M-1$-receiver DBC achievable region.

\section{BACKGROUND ON THE DBC}

The deterministic broadcast channel, illustrated in figure 1, has one sender and multiple receivers. The sender combines the $M$ independent messages $\left\{m_{j} \in\left\{1, \ldots, 2^{n R_{j}}\right\}\right\}_{j=1}^{M}$ to be sent to each receiver into a single length- $n$ string $\underline{x}=$ $\left(x_{1}, \ldots, x_{n}\right)^{\prime}$, where $x_{i} \in \mathcal{X}$. At receiver $j$ each symbol $y_{i}^{j} \in \mathcal{Y}_{j}$ is a deterministic function of $x_{i}$, i.e. $y_{i}^{j}=f_{j}\left(x_{i}\right)$. The $j$ th decoder attempts to reconstruct $m_{j}$, i.e. $\hat{m}_{j}=d_{j}\left(y^{j}\right)$. A memoryless probability distribution $P(X)$ on $x$, combined with $f_{1}, \ldots, f_{M}$, induces a memoryless joint distribution $P\left(Y^{1}, \ldots, Y^{M}\right)$ on $\left\{y^{1}, \ldots, y^{M}\right\}$. For a fixed memoryless $P(X)$, the set of all achievable rates $\mathcal{R}\left[P(X) ; f_{1}, f_{2}, \ldots, f_{M}\right]$ is given by [3], [2]

$$
\left\{\underline{R} \in \mathbb{R}_{+}^{M} \mid \sum_{i \in S} R_{i}<H(Y(S)) \forall S \subseteq\{1, \ldots, M\}\right\},
$$

where $Y(S)=\left\{Y^{j}, j \in S\right\}$. The capacity region of the DBC is given by

$\mathcal{R}\left[f_{1}, f_{2}, \ldots, f_{M}\right]=\mathrm{cl}\left[\mathrm{CH}\left(\bigcup_{P(X)} \mathcal{R}\left[P(X) ; f_{1}, f_{2}, \ldots, f_{M}\right]\right)\right]$

where $\mathrm{cl}$ denotes closure and $\mathrm{CH}$ denotes convex hull. 


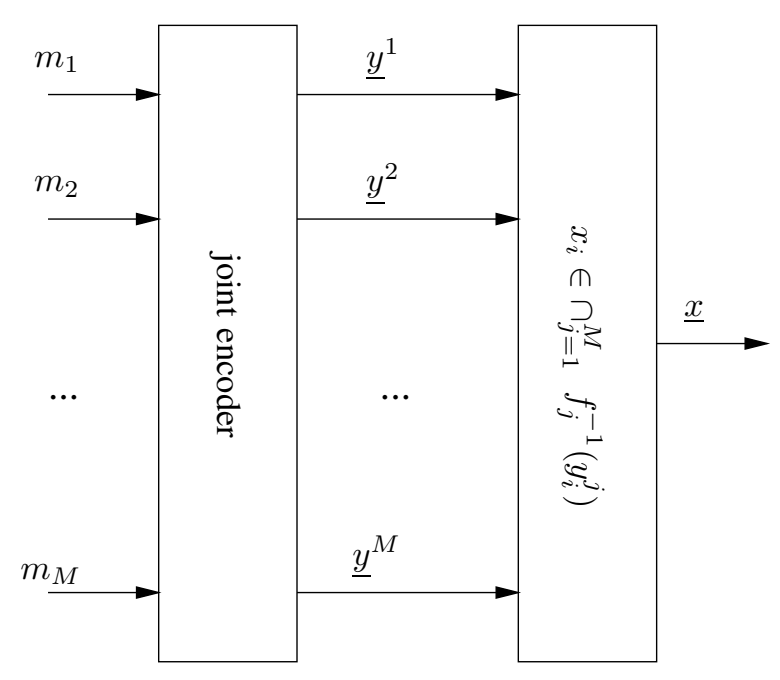

Fig. 2. Joint Encoding with Binning for the DBC.

\section{A. Binning as an Achievable Strategy}

As discussed in [4, sec. III], the dual relationship between the problems of SW and DBC manifests itself in the fact that the achievable rate strategies for the SW and DBC problems allow for one problem's encoder to mimic the other's decoder, and vice versa. Specifically, both achievability arguments use a binning approach, which partitions the set of all possible sequence outcomes, as their base. The SW encoder observes a sequence of symbols and specifies as its output the bin index associated with that sequence. This is parallel in operation of the DBC decoder. The SW decoder takes a set of messages that specify, for each source, the index of bin containing the source sequence. It then searches within these bins for a (unique with high probability) set of jointly typical sequences and specifies this as its output. Likewise, the DBC encoder takes a set of messages that specify, for each receiver, the index of the bin containing the received sequence. The DBC encoder next searches within these bins for a (non-unique with high probability) set of jointly typical sequences $\left(y^{1}, y^{2}, \ldots, \underline{y}^{M}\right)$. Having selected the output of the channel, the DBC encoder's final step is to choose an input sequence to produce this output. Specifically, for every $i \in\{1, \ldots, n\}$, the tuple $\left(y_{i}^{1}, y_{i}^{2}, \ldots, y_{i}^{M}\right)$ is used to select any $x_{i} \in \cap_{j=1}^{M} f_{j}^{-1}\left(y_{i}^{j}\right)$. Figure 2 illustrates this encoding strategy.

\section{B. Vertices: Successive Encoding}

If we consider an encoding strategy with a memoryless probability distribution $P(X)$, then the set of achievable rates $\mathcal{R}\left[P(X) ; f_{1}, f_{2}, \ldots, f_{M}\right]$ has 'vertices' or 'corner points' associated with expanding $H\left(Y^{1}, \ldots, Y^{M}\right)$ into $M$ terms by successive applications of the chain rule for entropy and assigning to each rate the unique corresponding term in the expansion. Transmitting at such rates allows for the joint search over all users' bins to be done successively. For example, consider communicating at the vertex rate $\left(R_{1}, R_{2}, \ldots, R_{M}\right)=$ $\left(H\left(Y^{1}\right), H\left(Y^{2} \mid Y^{1}\right), \ldots, H\left(Y^{M} \mid Y^{1} \ldots Y^{M-1}\right)\right)$ :

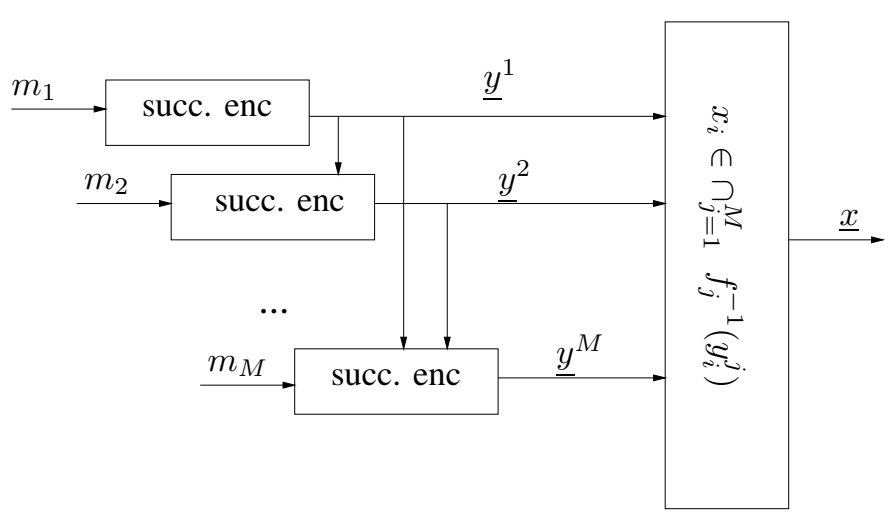

Fig. 3. Pipelined Encoder for Communicating at a Vertex Rate for the DBC.

- Encoding message $m_{1}$ at rate $R_{1}=H\left(Y^{1}\right)$ can be done by searching in the bin of message $m_{1}$ for a typical $\underline{y}^{1}$ sequence. This is because there are $2^{n R_{1}}$ such bins, one for each message $m_{1}$ index, and there are asymptotically $2^{n H\left(Y^{1}\right)}$ typical $y^{1}$ sequences.

- Consider any $j \in\{2, \ldots, M\}$. After successful encoding of $y^{1}, \ldots, y^{j-1}$, encoding message $m_{j}$ at rate $R_{j}=$ $H\left(\bar{Y}^{j} \mid Y^{1} \ldots Y^{j-1}\right)$ can be done by searching in the bin of message $m_{j}$ for a sequence $y^{j}$ that allows for $\left(\underline{y}^{1}, \ldots, \underline{y}^{j}\right)$ to be jointly typical. This is because there are $2^{n R_{j}}$ such bins, one for each message $m_{j}$ index, and there are asymptotically $2^{n H\left(Y^{j} \mid Y^{1} \ldots Y^{j-1}\right)}$ sequences $\underline{y}^{j}$ that allow for $\left(y^{1}, \ldots, y^{j}\right)$ to be jointly typical.

Figure 3 illustrates the successive encoding mechanism.

\section{RATE-SPLITTING FOR THE DBC}

We now illustrate that for a fixed memoryless distribution $P(X)$, a rate-splitting approach may be applied so that any rate in $\mathcal{R}\left[P(X) ; f_{1}, f_{2}, \ldots, f_{M}\right]$ may be transformed to a vertex in another $\mathcal{R}\left[P(X) ; \tilde{f}_{1 a}, \tilde{f}_{1 b}, \ldots, \tilde{f}_{(M-1) a}, \tilde{f}_{(M-1) b}, f_{M}\right]$, for some appropriately defined functions $\left\{\tilde{f}_{1 a}, \tilde{f}_{1 b}, \ldots, \tilde{f}_{(M-1) a}, \tilde{f}_{(M-1) b}\right\}$.

The dominant face $\mathcal{D}\left[\mathcal{R}\left[P(X) ; f_{1}, f_{2}, \ldots, f_{M}\right]\right]$ is given by

$\left\{R \in \mathcal{R}\left[P(X) ; f_{1}, f_{2}, \ldots, f_{M}\right] \mid \sum_{i=1}^{M} R_{i}=H\left(Y^{1}, \ldots Y^{M}\right)\right\}$.

Since any point in $\mathcal{R}\left[P(X) ; f_{1}, f_{2}, \ldots, f_{M}\right]$ is dominated (with respect to the standard partial order on $\mathbb{R}_{+}^{M}$ ) by a point in $\mathcal{D}\left[\mathcal{R}\left[P(X) ; f_{1}, f_{2}, \ldots, f_{M}\right]\right]$, we restrict our attention to rates lying on $\mathcal{D}\left[\mathcal{R}\left[P(X) ; f_{1}, f_{2}, \ldots, f_{M}\right]\right]$.

We now discuss the two-receiver problem where $m_{1} \in$ $\left\{1, \ldots, 2^{n R_{1}}\right\}, \quad m_{2} \in\left\{1, \ldots, 2^{n R_{2}}\right\}$ and $\left(R_{1}, R_{2}\right) \in$ $\mathcal{D}\left[\mathcal{R}\left[P(X) ; f_{1}, f_{2}\right]\right]$ but $\left(R_{1}, R_{2}\right)$ is not a vertex. Consider the probability distribution $P\left(Y^{1}, Y^{2}\right)$ induced by $P(X)$ and $f_{1}, f_{2}$. Perform a splitting operation to construct $Y_{i}^{1 a}=$ $g_{1 a}\left(Y_{i}^{1}\right), Y_{i}^{1 b}=g_{1 b}\left(Y_{i}^{1}\right)$ such that $\left(Y_{i}^{1 a}, Y_{i}^{1 b}\right)$ and $Y_{i}^{1}$ form a bijection. In other words, construct a set of three functions

$$
g_{1 a}: \mathcal{Y}_{1} \rightarrow \mathcal{Y}_{1}, g_{1 b}: \mathcal{Y}_{1} \rightarrow \mathcal{Y}_{1}, g_{1}: \mathcal{Y}_{1} \times \mathcal{Y}_{1} \rightarrow \mathcal{Y}_{1}
$$


such that for all $y \in \mathcal{Y}_{1}$,

$$
g_{1}\left(g_{1 a}(y), g_{1 b}(y)\right)=y .
$$

Constructions of $\left\{g_{1 a}, g_{1 b}, g_{1}\right\}$ can be found in [12], [13], [14]. 'Source-splitting' for Slepian-Wolf was introduced in [12], but the technique given there requires common sources of randomness to be shared between the encoders and the decoder. Moreover, in that approach, the alphabet size of the outputs of the splitter has cardinality greater than that of the original source. Another approach in [13], [14] assumes without loss of generality that $\mathcal{Y}_{1}=\{0,1, \ldots, Q-1\}$. From there the splitting mechanism is constructed as follows:

$$
\begin{aligned}
g_{1 a}\left(y^{1}\right) & =\min \left(\pi\left(y^{1}\right), T\right) \\
g_{1 b}\left(y^{1}\right) & =\max \left(\pi\left(y^{1}\right), T\right)-T \\
g_{1}\left(y^{1 a}, y^{1 b}\right) & =\pi^{-1}\left(y^{1 a}+y^{1 b}\right)
\end{aligned}
$$

where $T \in \mathcal{Y}_{1}$ and $\pi$ is a permutation of $\mathcal{Y}_{1}$. Such a splitting mechanism induces a memoryless distribution $P\left(Y^{1 a}, Y^{1 b}, Y^{2}\right)$ where $H\left(Y^{1 a}, Y^{1 b}, Y^{2}\right)=H\left(Y^{1}, Y^{2}\right)$. As discussed in [14, sec. 3.1], splitting according to (1) allows for any rate $R \in \mathcal{D}\left[\mathcal{R}\left[P(X) ; f_{1}, f_{2}\right]\right]$ to satisfy

$$
\begin{aligned}
R_{1 a} & =H\left(Y^{1 a}\right) \\
R_{2} & =H\left(Y^{2} \mid Y^{1 a}\right) \\
R_{1 b} & =H\left(Y^{1 b} \mid Y^{1 a}, Y^{2}\right) \\
R_{1} & =R_{1 a}+R_{1 b} .
\end{aligned}
$$

Now let us assume the splitting operation has been performed so that (2) holds. The encoder takes the message $m_{1} \in$ $\left\{1, \ldots, 2^{n R_{1}}\right\}$ and represents it as a pair of messages

$$
\begin{gathered}
m_{1} \in\left\{1, \ldots, 2^{n R_{1}}\right\} \\
\Leftrightarrow \\
\left(m_{1 a}, m_{2 a}\right) \in\left(\left\{1, \ldots, 2^{n R_{1 a}}\right\},\left\{1, \ldots, 2^{n R_{1 b}}\right\}\right) .
\end{gathered}
$$

It partitions all possible $y^{1 a}$ sequences into $2^{n R_{1 a}}$ bins, all possible $y^{1 b}$ sequences into $2^{n R_{1 b}}$ bins, and all $\underline{y}^{2}$ sequences into $2^{n R_{2}^{-}}$bins. Encoding is done as if to construct a jointly typical $\left(\underline{y}^{1 a}, \underline{y}^{1 b}, \underline{y}^{2}\right)$. Note that although $\left(R_{1}, R_{2}\right)$ is not a vertex in $\mathcal{R}\left[\bar{P}(X) ; f_{1}, f_{2}\right],\left(R_{1 a}, R_{1 b}, R_{2}\right)$ is indeed a vertex in $\mathcal{R}\left[P(X) ; g_{1 a} \circ f_{1}, g_{1 b} \circ f_{1}, f_{2}\right]$; thus the encoding strategy described in section II-B suffices. See figure 4. The decoder for receiver 2 observes $\underline{y}^{2}$ and specifies its bin number $m_{2}$. Receiver 1's decoder observes $y^{1}$, performs the splitting operation to construct $\left(y^{1 a}, y^{1 b}\right)$, and specifies the bin numbers $\left(m_{1 a}, m_{1 b}\right)$. Finally, the message pair $\left(m_{1 a}, m_{2 a}\right)$ is combined to form the message $m_{1}$ by simply reversing the operation (3). See figure 5. This approach generalizes to $M$ users.

Theorem 3.1: Any achievable rate for an arbitrary $M$ receiver $\mathrm{DBC}$ can be attained via rate-splitting towards a $(2 M-1)$-receiver DBC, where each user is split at most once and successive encoding suffices.

Since $\mathcal{D}\left[\mathcal{R}\left[P(X) ; f_{1}, f_{2}, \ldots, f_{M}\right]\right]$ has precisely the same characterization as the dominant face of the $M$-source SlepianWolf achievable rate region, the proof of theorem 3.1 follows from the analogous source-splitting results for the SlepianWolf problem [14, sec. 3.2-3.3].

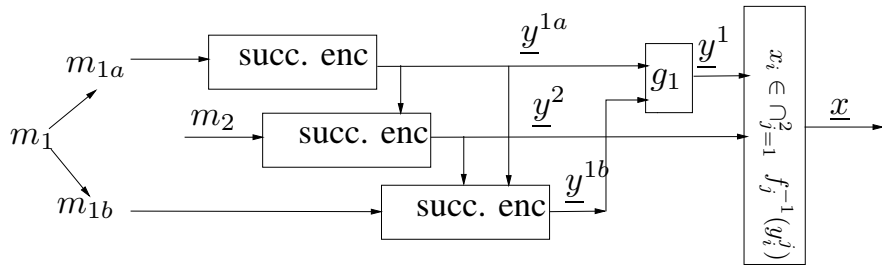

Fig. 4. Rate-splitting Based Encoding for the DBC.

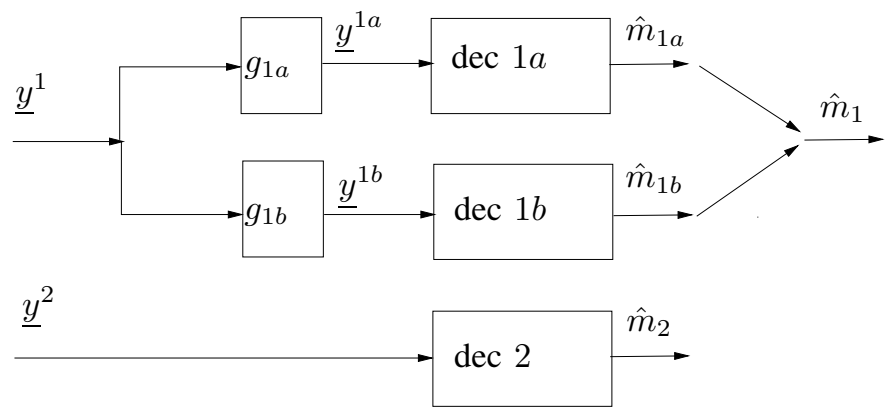

Fig. 5. Rate-Splitting Based Decoding for the DBC.

\section{CONCLUSION}

In this paper we have shown how the deterministic broadcast channel also exhibits a rate-splitting property. This implies that implementing a pipelined set of single-receiver DBC encoding with side information problems suffices to achieve any rate in the capacity region. This amounts to significant complexity reduction.

We further plan to construct a general-purpose lowcomplexity single-receiver DBC encoding with side information implementation so that, by combining this with ratesplitting, any instantiation of the DBC problem may be addressed practically. We also would like to explore how appropriate DBC models can touch upon multiterminal wireless communication. A first step in that direction is discussed in [16], where we develop capacity-achieving codes and lowcomplexity encoding/decoding algorithms for DBCs addressing wireless interference management.

\section{REFERENCES}

[1] D. Slepian and J. K. Wolf, "Noiseless coding of correlated information sources," IEEE Transactions on Information Theory, vol. 19, no. 4, pp. 471-480, 1973.

[2] K. Marton, "The capacity region of deterministic broadcast channels," in IEEE International Symposium on Information Theory, Paris-Cachan, France, 1977.

[3] M. S. Pinsker, "Capacity of noiseless broadcast channels," Probl. Inform. Transm., pp. 97-102, 1978.

[4] T. M. Cover, "Comments on broadcast channels," IEEE Transactions on Information Theory, vol. 44, pp. 2524-2530, 1998.

[5] H. Liao, Multiple Access Channels, $\mathrm{PhD}$ dissertation, University of Hawaii, Department of Electrical Engineering and Computer Science, June 1972.

[6] R. Ahlswede, "Multi-way communication channels," ISIT, pp. 23-52, 1971.

[7] N. Jindal, S. Vishwanath, and A. Goldsmith, "On the duality between general multiple-access/broadcast channels," in IEEE International Symposium on Information Theory, Yokohama, Japan, June 29-July 4 2003. 
[8] T. M. Cover and J. Thomas, Elements of Information Theory, John Wiley \& Sons, New York, NY, 1991.

[9] S. C. Draper, B. Frey, and F. Kschischang, "On interacting encoders and decoders in multiuser settings," in IEEE International Symposium on Information Theory, Chicaco , Ill, June 27-July 22004.

[10] V. Stankovic, S. Cheng, and Z. Xiong, "On dualities in multiterminal coding problems," in Proc. 42nd Annual Allerton Conference on Communications, Control and Computing, Monticello, IL, October 2004.

[11] A. Grant, B. Rimoldi, R. Urbanke, and P. A. Whiting, "Rate-splitting multiple access for discrete memoryless channels," IEEE Transactions on Information Theory, vol. 47, no. 3, pp. 873-890, 2001.

[12] B. Rimoldi and R. Urbanke, "Asynchronous Slepian-Wolf coding via source-splitting," in IEEE International Symposium on Information Theory, Ulm, Germany, June 29-July 4 1997, p. 271.

[13] T. P. Coleman, A. H. Lee, M. Médard, and M. Effros, "On some new approaches to practical Slepian-Wolf compression inspired by channel coding," in IEEE Data Compression Conference, Snowbird, Utah, March 23 - March 252004.

[14] T. P. Coleman, A. H. Lee, M. Médard, and M. Effros, "Low-complexity approaches to Slepian-Wolf near-lossless distributed data compression," IEEE Transactions on Information Theory, 2004, submitted for review, October 2004

[15] A. D. Wyner, "On source coding with side information at the decoder," IEEE Transactions on Information Theory, vol. 21, pp. 294-300, 1975.

[16] T. P. Coleman, E. Martinian, M. Effros, and M. Medard, "Interference management via capacity-achieving codes for the deterministic broadcast channel," in IEEE Information Theory Workshop on Coding and Complexity, Rotorua, New Zealand, August 2005. 